\title{
Análise de variações no desempeno lumínico do Centro Cívico de Curitiba através de modelagem e simulação paramétrica
}

Analysis of variations in daylight performance of the Curitiba Civic Center through parametric modeling and simulation

\author{
Ernesto Bueno \\ Universidade Positivo, Brasil \\ ernestobueno@gmail.com
}

\author{
Antônio Carlos de Quadros Gonçalves Neto \\ Universidade Positivo, Brasil \\ antoniodequadros@outlook.com
}

\author{
Caio Henrique Mehl \\ Universidade Positivo, Brasil \\ caiomehl2@hotmail.com
}

\begin{abstract}
To ensure access to sunlight in urban planning, specialized software is available. Mainly used in the initial stages, these tools allow the study of the environmental performance of the proposal. However, neighborhood impact is seen as a secondary aspect, usually evaluated with GIS tools, simulating pre-existing or proposed situations. However, visual programming tools allow, data processing in addition to parametric modeling, streamlining the process of analysis of architectural and urban pre-existences and proposals. From a case study, we present a methodology that uses these tools to demonstrate the loss of daylight performance of open spaces due to urban densification.
\end{abstract}

Keywords: Urban daylight performance; Environmental performance simulation; Parametric urban modeling; Grasshopper.

\section{Introdução}

A modelagem paramétrica, através de ferramentas de programação visual como Grasshopper, mostra competências no desenvolvimento de projetos sistêmicos e arrojados de arquitetura e urbanismo contemporâneos (Bueno, 2016). Já as ferramentas de processamento de informação tais como GIS permitem, dentre outras opções, criar simulações de situações existentes ou propostas para análises quantitativas do que se estuda. Considerando que ferramentas de programação visual permitem o processamento de dados além da modelagem paramétrica (Davis \& Peters, 2013), é possível o emprego deste tipo de ferramentas para o processamento e simulação de informações de planejamento urbano, ultrapassando algumas limitações do GIS (Roudsari \& Pak, 2013).

Fahmy e Elwy (2016) exploram este tipo de configuração metodológica para encontrar relações entre adensamento urbano e conforto térmico. Roudsari e Pak (2013) desenvolveram uma serie de plug-ins que potencializa este modo de trabalhar, conectando mais eficientemente a modelagem paramétrica com a avaliação de desempenho ambiental, o que é demonstrado através do estudo de modelos genéricos. Porém, ambos utilizam desta técnica para analisar cenários urbanos e arquitetônicos hipotéticos. Sem a inclusão destes métodos de simulação de desempenho ambiental no planejamento urbano em regiões em desenvolvimento, os urbanistas não conseguem relacionar critérios de adensamento a variações deste tipo de desempenho dos espaços urbanos com suficiente agilidade, estagnando a capacidade de apresentar planos diretores resilientes às mudanças das cidades.
No presente trabalho, utilizamos destas ferramentas para analisar uma região urbana existente e já consolidada. Estuda-se a Avenida Cândido de Abreu, no Centro Cívico de Curitiba, Brasil, no decorrer de 32 anos, com três recortes temporais que demonstram diferenciações entre os tipos de ocupação e verticalização da região: 1985, 1997 e 2017. A pesquisa visa contribuir com a inclusão de métodos paramétricos de simulação que podem agilizar processos de planejamento urbano ao permitir a definição de padrões de ocupação adensada, com bom desempenho lumínico nos espaços urbanos. Parte da metodologia utilizada baseia-se em Bueno e Patron (2014).

Neste contexto, utilizamos o termo desempenho lumínico para englobar o desempenho de iluminação natural e de insolação, conceitos revisados em Scalco, Pereira e Rigatti (2010).

\section{Revisão da literatura}

Na procura de uma relação entre as alturas dos edifícios e a iluminação natural, $\mathrm{Ng}$ (2005) foca na modelagem simplificada de uma área hipotética, seguindo a legislação urbanística de Hong Kong, utilizando o FormZ; e Autodesk Lightscape para medir o fator de luz diurna vertical. Demonstrou que a variação de alturas pode melhorar o desempenho de iluminação em pavimentos inferiores.

Araújo e Cabús (2007) estudam a luz refletida para iluminação em cânions urbanos, áreas com dificuldade de acesso de luz. A metodologia usada compara a intensidade da luz direta e da luz refletida, incluindo a radiação solar, obtidas com o uso do software TropLux. Os resultados mostram que a influência da luz refletida é grande, e que é necessária uma atenção especial às refletâncias do entorno e à orientação das 
aberturas e das obstruções para a melhor iluminação natural nos ambientes internos.

Scalco, Pereira e Rigatti (2010), desenvolveram um método para a avaliação dos impactos de novas edificações em vizinhanças urbanas com relação ao acesso às condições mínimas de insolação e iluminação natural. Modelaram um cenário atual e um cenário de ocupação máxima prevista pela legislação. Validam a metodologia, mesmo não obtendo variações significativas de impacto, cujo objeto é uma edificação específica, e não os espaços urbanos da vizinhança.

Leidi e Schlüter (2013) propõem uma metodologia de análise, visualização e extração de dados a serem utilizados no desenvolvimento de um projeto arquitetônico no contexto urbano. Eles desenvolveram um software em Processing para aplicar esta metodologia. Com isto obtêm-se dados sobre radiação solar, ventilação, visibilidade etc., discretizados num grid tridimensional. O potencial aproveitamento está no estudo de massas de edificações a ocupar o espaço estudado, e etapas posteriores relacionadas com o invólucro.

Com um enfoque em conforto térmico e visual de espaços externos em zonas áridas, Fahmy e Elwy (2016) exploram a influência das diferentes alturas e orientações das edificações no resfriamento passivo de áreas de circulação e permanência de pedestres. O objetivo era encontrar uma forma urbana satisfatória que ofereça o mínimo de calor e boa iluminação natural para ambos os espaços internos e externos, o que pode contribuir para o planejamento urbano. Para tal, o software Rhinoceros e seu plug-in, Grasshopper, com as extensões HoneyBee e LadyBug foram usados. Um modelo tridimensional simplificado de um cenário hipotético foi criado e analisado com as condicionantes climáticas de regiões áridas.

Verifica-se na literatura estudada, que o objeto de estudo é a desempenho edificação em função dos seus espaços internos. A influência dos vazios urbanos, mesmo importante para o cálculo do desempenho, fica num segundo plano. Esta falta de foco prejudica a inclusão de este tipo de conhecimento nas discussões sobre a qualidade dos espaços públicos face à densificação urbana.

\section{Procedimentos metodológicos}

\section{Escolha dos softwares para análise}

A busca por metodologias previamente executadas ofereceram três softwares para construção da análise. Troplux (Araújo \& Cabús, 2007) é ideal para medições de iluminância em ambientes internos, mas, assim como o software Dialux, não é apropriado para a análise de iluminâncias de um grande espaço externo. Autodesk Ecotect mensura diversos fatores de conforto ambiental em áreas externas, porém a modelagem 3D depende de outros softwares, e é a tarefa que mais influência nos resultados obtidos (Breviglieri \& Amaral, 2011). Rhinoceros, utilizado por Fahmy e Elwy (2016) para modelagem 3D, é capaz de receber uma variedade de plugins, dentre eles, o Grasshopper, uma ferramenta de modelagem paramétrica que utiliza linguagem gráfica de programação.

Para análises simplificadas, os dois softwares conseguem calcular fatores ambientais como impacto de sombras (Bueno \& Patron, 2014). Para cálculos mais complexos é necessário adicionar algum plug-in para uma análise mais específica. $O$ LadyBug possui capacidade de gerar dados e análises para Rhino/Grasshopper, além de permitir feedback quase instantâneo em todas as interações (Roudsari \& Pak, 2013). Ele permite importar e analisar dados meteorológicos padrões no Grasshopper; gerar diagramas; executar análise de radiação, estudos de sombra e análise de visuais.

O Honeybee conecta o Grasshopper a ferramentas de análise, como EnergyPlus, Radiance, Daysim e OpenStudio, para a verificação de performance de energia, conforto, iluminação natural (ibid.). Estes são capazes de construir análises em ambientes internos e externos, porém não se aplicam a pesquisa, pois LadyBug e Honeybee não realizam leitura de referente à iluminância nos espaços públicos, o qual é tema central desta pesquisa.

Considerando o exposto, o plug-in utilizado para desenvolver a pesquisa e gerar os dados foi o DIVA for Rhino, por ser eficiente em otimizar e combinar cálculos detalhados referente às performances da luz solar e ao acesso a iluminação natural e suas variáveis, abrangendo desde o impacto de sobras até a propriedade dos materiais do ambiente a ser analisado (González \& Fiorito, 2015).

\section{Modelagem dos cenários}

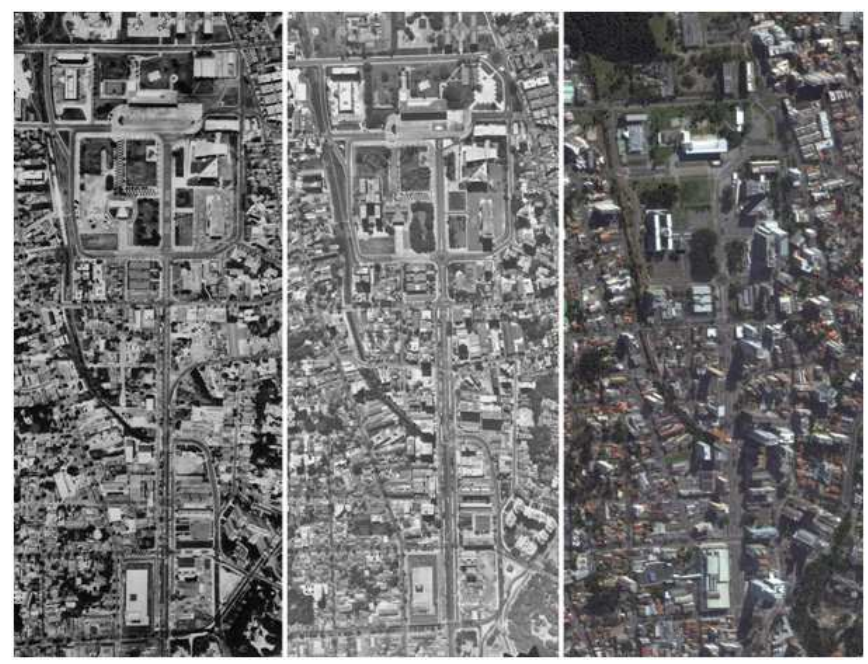

Figura 1: Recorte das ortofotos da área de estudo na Av. Cândido de Abreu, Curitiba (COMEC, 1985); (IPPUC/TELEPAR, 1997); e (COPEL/SANEPAR, 2017).

Após a escolha dos métodos de análise e softwares, foi realizado um levantamento in-loco e por ortofotos da área (Figura 1). Deste levantamento se obtiveram as dimensões das edificações, dados que logo foram contrastados com os desenhos dos polígonos de contorno destas edificações na base cartográfica. As informações planimétricas da Avenida em formato DWG e ortofotos foram fornecidas pelo IPPUC (Instituto de Pesquisa e Planejamento Urbano de Curitiba). 
Outra fonte de dados acessada para a modelagem foi o 3D Warehouse (http://3dwarehouse.sketchup.com), o armazém de modelos de SketchUp de acesso livre, que inclui modelos de edificações reais compartilhados por usuários (sistema que suportava a visualização 3D da versão anterior do Google Earth) seguido da modelagem tridimensional. Com todas as linhas importadas para o Rhinoceros, a modelagem foi feita de forma paramétrica através do Grasshopper, interpolando as curvas de nível para gerar uma topografia precisa do local. As linhas e polígonos importados da base cartográfica foram usados para dividir a superfície topográfica em quadras, ruas e calçadas. A maioria dos edifícios foram extrudados dos seus contornos 2D a partir da superfície das quadras e tiveram suas alturas estabelecidas pelo levantamento in-loco. Alguns edifícios significativos foram modelados manualmente no Rhino, comparando os dados levantados com os modelos do 3D Warehouse, resultando em sólidos mais detalhados do que as simples extrusões.

\section{Simulações de desempenho lumínico}

Após a geração e ajustes dos modelos, que naturalmente são polissuperfícies NURBS, passa-se à geração de malhas poligonais de análise. Os cálculos de desempenho lumínico são efetuados relacionando vetores de incidência solar com os vértices, faces e normais das malhas poligonais, razão pela qual é necessária uma discretização que, sem modificar a geometria, aumenta a densidade da informação. Quanto mais densa for a malha, melhor resolução terá a análise. Porém, quanto maior for a resolução requerida, maior será o tempo de execução e a carga de memória do computador, pudendo inviabilizar simulações em escala urbana (Bueno \& Turkienicz, 2014). Para evitar este problema, o processo geração de malhas poligonais foi feita no Grasshopper com diferentes aproximações, dependendo da parte do modelo: (i) a superfície do terreno; (ii) as edificações extrudadas e de faces retangulares; e (iii) as edificações significativas, de formas complexas.

A superfície do terreno interpolada foi subdividida na região central, foco da análise, e o entorno, região de menor resolução. Em ambas se utilizou o componente Mesh Surface. A região central teve uma largura aproximada de $80 \mathrm{~m}$ e foi discretizada com um valor de amostragem inicial de $3 \mathrm{~m}$. Considerando o arredondamento da interpolação e acidentes geográficos, as novas faces ficaram com uma largura, em média, em torno de $2,96 \mathrm{~m}$. Na região do entorno, a amostragem foi de $9 \mathrm{~m}$.

As edificações extrudadas e de faces retangulares foram subdivididas em superfícies e nelas aplicado o mesmo critério do terreno de análise. Também se usou uma referência de amostragem de $3 \mathrm{~m}$. Nas edificações significativas, de formas complexas (ou de faces de mais de 4 lados), se utilizou o componente Mesh Brep, configurando o parâmetro de distância máxima de subface com o mesmo valor de amostragem.

Para estas simulações foram escolhidos dois momentos representativos dos extremos de conforto durante o ano: 0 solstício de inverno (Figura 4) e o solstício de verão (Figura 5).

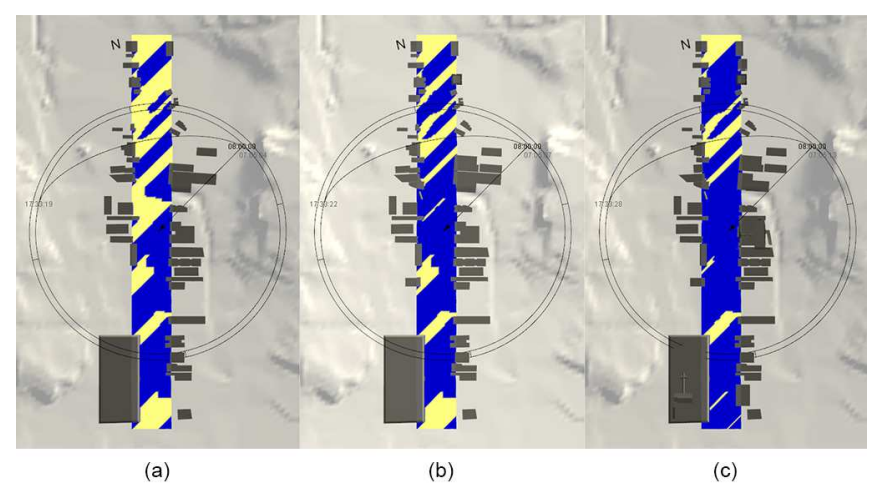

Figura 2: Plantas da simulação de impacto de sombras às $08: 00 \mathrm{~h}$ do solstício de inverno de (a) 1985, (b) 1997 e (c) 2017.

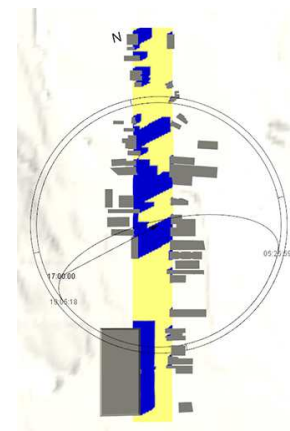

(a)

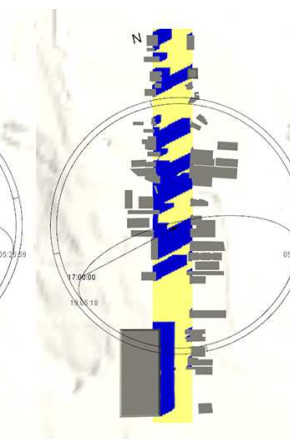

(b)

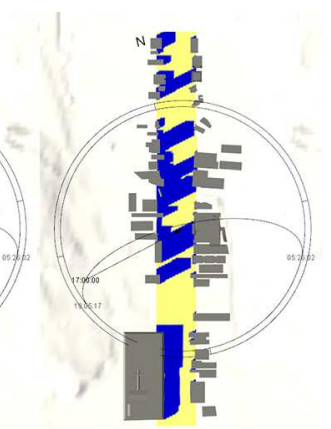

(c)
Figura 3: Plantas da simulação de impacto de sombras às 17:00 h do solstício de verão de (a) 1985, (b) 1997 e (c) 2017.

As simulações de impacto de sombras foram desenvolvidas utilizando componentes de Grasshopper, adicionando o script de posição solar atualizado em Vannini, Bueno e Turkienicz (2012), e Bueno e Patron (2014), a partir da implementação em VB.NET de Ted Ngai, do algoritmo de posição solar da Solar Radiation Monitoring Laboratory da Universidade de Oregon

(http://solardat.uoregon.edu/SolarRadiationBasics.html). Ao executar o script, se gera o vetor do sol para o momento definido. Este vetor é usado como parâmetro de entrada no componente Exposure para computar a exposição da luz direta sobre a malha de análise, considerando as edificações como objetos de oclusão. Os valores de oclusão-exposição, que são normalizados (0 para oclusão e 1 para exposição, para cada vértice da malha), são contabilizados e, com estes, calculada a porcentagem de sombra sobre a superfície de estudo. Esta mensuração foi feita multiplicando o valor de oclusão-exposição (0 ou 1), vezes a área de cada face da malha de análise; fazendo a somatória de áreas de faces expostas; e calculando a porcentagem de área oclusa em relação à área total da malha de análise. Complementariamente, se geram plantas para cada cenário, (apresentadas na Figura 2 e Figura 3), com as quais é possível estabelecer graficamente a comparação. 


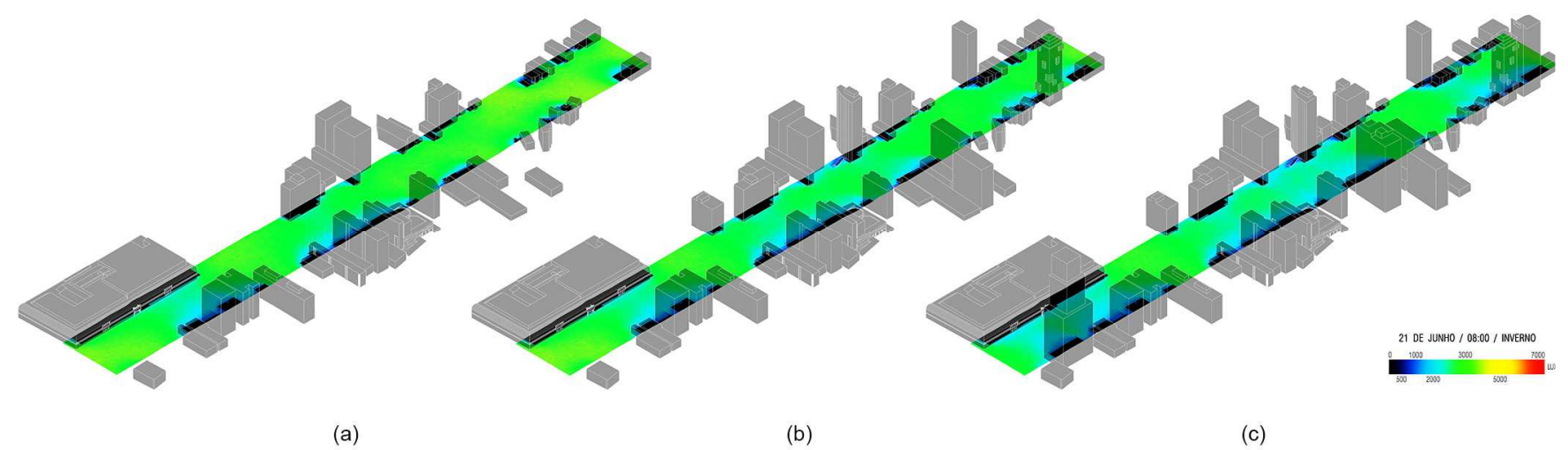

Figura 4: Isometrias da simulação de iluminância às 8:00 h do solstício de inverno de (a) 1985, (b) 1997 e (c) 2017.

Pelas limitações de implementação de cálculos de iluminância diretamente no Grasshopper e nas outras ferramentas previamente mencionadas, para as simulações deste tipo de desempenho foi usado o DIVA. Utilizando componentes deste plug-in, foi conectado o modelo do Grasshopper com as análises que o DIVA executa (através do Daysim), retornando os resultados em lux de volta ao Grasshopper. Entretanto o plug-in foi desenvolvido visando analisar espaços internos, tornando assim a classificação dos intervalos de iluminância incompatível com espaços externos, pois o plug-in atribui 2 mil Ix como uma região com alta taxa de iluminância, o que está certo para ambientes internos, mas para externos é considerado média, assim foi utilizada a ferramenta Gradient do Grasshopper para mudar essa classificação então atribuir 2 mil Ix como médio percentual de iluminância e 7 mil como alto, podendo assim gerar as imagens com intervalos corretos dos valores e cores (Figura 4 e Figura 5).

\section{Resultados}

Os valores numéricos resultantes das análises estão resumidos na Tabela 1, para os cenários de inverno, e na Tabela 2, para os cenários de verão. Comparando os valores, podemos perceber que a porcentagem de sombreamento no inverno tem aumentado um 20,22\% entre 1985 e 1997, e um $14,65 \%$ entre 1997 e 2017 . Isto significa que a porcentagem de área ensolarada tem diminuído proporcionalmente.
Tabela 1: Resumo de valores de desempenho lumínico simulados para as 8:00 h do solstício de inverno, dos anos estudados.

\begin{tabular}{|llll|} 
Cenário & $\begin{array}{l}\text { Sombreamento } \\
(\%)\end{array}$ & $\begin{array}{l}\text { Iluminância } \\
\text { Média }(\mathrm{Ix})\end{array}$ & $\begin{array}{l}\text { Iluminância } \\
\text { Máxima }(\mathrm{Ix})\end{array}$ \\
\hline Inverno, 1985 & 61,21 & 2925 & 4003 \\
\hline Inverno, 1997 & 73,59 & 2448 & 3902 \\
\hline Inverno, 2017 & 84,37 & 2301 & 3401 \\
\hline
\end{tabular}

Tabela 2: Resumo de valores de desempenho lumínico simulados para as 17:00 h do solstício de verão, dos anos estudados.

\begin{tabular}{|llll|}
\hline Cenário & $\begin{array}{l}\text { Sombreamento } \\
(\%)\end{array}$ & $\begin{array}{l}\text { Iluminância } \\
\text { Média }(\mathrm{Ix})\end{array}$ & $\begin{array}{l}\text { Iluminância } \\
\text { Máxima (Ix) }\end{array}$ \\
\hline Verão, 1985 & 43,38 & 4225 & 7103 \\
\hline Verão, 1997 & 50,93 & 3848 & 6502 \\
\hline Verão, 2017 & 54,26 & 3701 & 6201 \\
\hline
\end{tabular}

Ainda no inverno, acompanhando a variação de iluminância, também podemos perceber uma diminuição. A iluminância média diminuiu um 16,31\% entre 1985 e 1997, e um $6 \%$ entre 1997 e 2017. A iluminância máxima diminuiu um $2,52 \%$ entre 1985 e 1997, e um 12,84\% entre 1997 e 2017.

A porcentagem de sombreamento no verão tem aumentado um 17,4\% entre 1985 e 1997, e um 6,54\% entre 1997 e 2017,

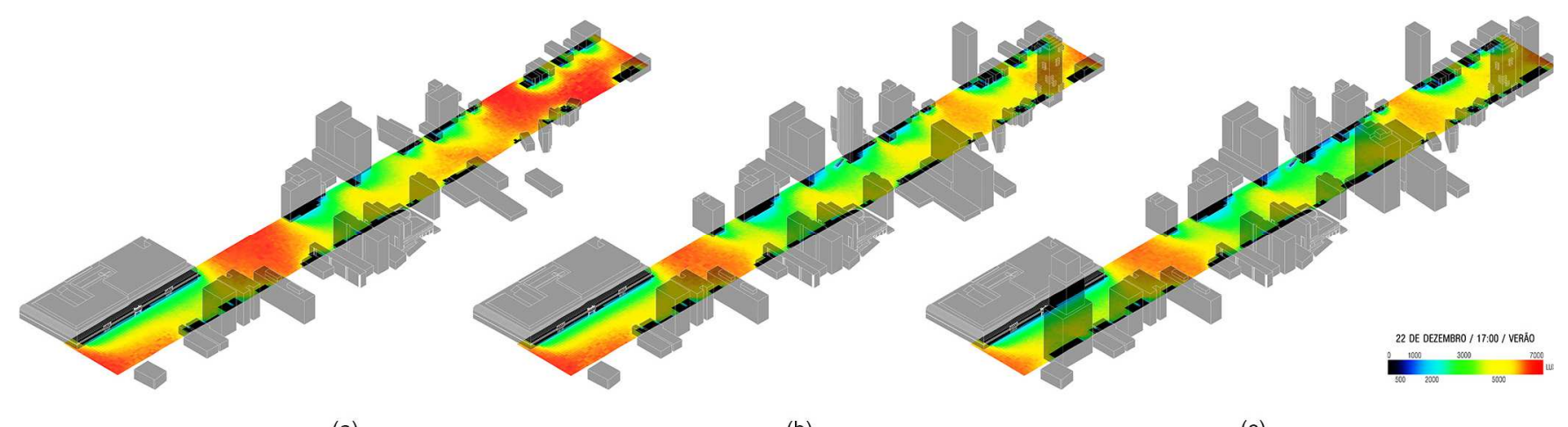

(a)

(b)

(c)

Figura 5: Isometrias da simulação de iluminância às 17:00 h do solstício de verão de (a) 1985, (b) 1997 e (c) 2017. 
diminuído proporcionalmente a porcentagem de área ensolarada.

Nas variações de iluminância no verão, vemos que a iluminância média diminuiu um 8,92\% entre 1985 e 1997, e um 3,82\% entre 1997 e 2017. A iluminância máxima diminuiu um 8,46\% entre 1985 e 1997, e um 4,63\% entre 1997 e 2017.

Isso comprova que o impacto é maior no inverno, no momento em que é mais crítica a necessidade de acesso ao sol, com conforto lumínico e grande influência no conforto térmico.

\section{Discussão}

Os resultados nos permitem questionar as normativas de planejamento urbano e demonstrar que, mesmo seguindo a legislação, a região de estudo apresenta áreas com deficiência de luz solar direta e indireta, prejudicando assim o uso dos espaços públicos. Mediante as comparações entre os cenários selecionados, constata-se quais espaços da área de estudo foram prejudicados ou beneficiados pelas novas edificações; quais regiões da Avenida sofreram maior impacto devido aos novos empreendimentos. Com estudos continuados desta forma, será possível identificar padrões de ocupação que causam maior e menor impacto.

É sabido que a legislação brasileira possui uma influência significativa sobre o conforto térmico das edificações (Poehls, 2012). Mesmo que o conhecimento sobre este tema se concentre principalmente em espaços internos, podemos interpretar que essa afirmação se aplica também aos espaços públicos. Por esta razão é de vital importância a atualização das metodologias de planejamento, aproveitando melhor a evolução das tecnologias digitais em prol de projetos urbanos resilientes às mudanças das cidades.

\section{Agradecimentos}

Esta pesquisa foi desenvolvida a partir do projeto: Análise das variações de iluminância e sombreamento nos espaços da Av. Cândido de Abreu, Curitiba, relacionados ao adensamento urbano entre 1985 e 2017, dentro do Programa de Iniciação Científica da Universidade Positivo (www.up.edu.br/pic).

\section{Referências}

Araújo, I. A. L.; \& Cabús, R. C. (2007). Influência da luz natural refletida pelo entorno na iluminação de edifícios em cânions urbanos no trópico úmido. IX Encontro nacional e $\mathrm{V}$ latino americano de Conforto no Ambiente Construído. (pp. 86-95) Ouro Preto, MG.

Breviglieri, E. P. C., \& Amaral, J. S. J. (2011). 2․ Simpósio Brasileiro de Qualidade do Projeto no Ambiente Construído. X Workshop
Brasileiro de Gestão do Processo de Projeto na Construção de Edifícios. Rio de Janeiro, Brasil.

Bueno, Ernesto. (2016). Grasshopper. In: F. Braida, \& F. Lima, et al. (Eds.), 101 Conceitos da Arquitetura e Urbanismo na Era Digital (pp. 152-156). São Paulo: ProBooks.

Bueno, E., \& Patron, R. (2014). Um marco de trabalho para a simulação do passado arquitetônico como entendimento para a construção do futuro. Anais do $6^{0}$ Seminário Internacional de Curitiba. Curitiba: Universidade Positivo.

Bueno, E. \& Turkienicz, B., (2014). Supporting tools for early stages of architectural design. International Journal of Architectural Computing, 12(4). doi: 10.1260/1478-0771.12.4.495

Davis, D., \& Peters, B. (2013). Design Ecosystem: Customising the Architectural Design Environment With Software Plug-ins. Architectural Design, 83(2), 124-131. doi: 10.1002/ad.1567

Fahmy, M., \& Elwy, I. (2016). Visual and Thermal Comfort Optimization for Arid Urban Spaces using Parametric Techniques on the Scale of Compactness Degree. 36th International Conference on Pasive and Low Energy Architecture (pp. 10511059). Los Angeles: PLEA.

González, J., \& Fiorito, F. (2015). Daylight Design of Office Buildings: Optimisation of External Solar Shadings by Using Combined Simulation Methods. Buildings, 5(2), 560-580; doi: 10.3390/buildings5020560

Leidi, M., \& Schlüter, A. (2013). Exploring Urban Space Volumetric Site Analysis for Conceptual Design in the Urban Context. International Journal of Architectural Computing, 11(2). doi: 10.1260/1478-0771.11.2.157

$\mathrm{Ng}$, Edward. (2005). Study of the Relationship Between Daylight Performance and Height Difference of Building in High Density Cities Using Computational Simulation. 9th International IBPSA Conference (pp. 847-852). Montreal, Canada.

Poehls, Lennart. (2012). Avaliação da legislação brasileira como influência sobre o conforto térmico de edifícios para escritórios em Porto Alegre. (Dissertação de Mestrado, Universidade Federal do Rio Grande do Sul, Porto Alegre). Retrieved from http://hdl.handle.net/10183/61442

Roudsari, M., \& Pak, M. (2013). Ladybug: A parametric environmental plugin for Grasshopper to help designers create an environmentally-conscious design. Proceedings of BS2013: 13th Conference of International Building Performance Simulation Association (pp. 3128-3135). Chambéry, France.

Scalco, V. A., Pereira, F. O. R., \& Rigatti, D. (2010). Impacto de novas edificações na vizinhança: Proposta de método para a análise das condições de iluminação natural e de insolação. Ambiente Construído, 10(2). doi: 10.1590/S1678-86212010000200012

Vannini, V; Bueno, E; Turkienicz, B. (2012) Otimização geométrica de superfícies de fachada para uso fotovoltaico. SIGraDi 2012 [Proceedings of the 16th Iberoamerican Congress of Digital Graphics] Brasil - Fortaleza 13-16 November 2012, pp. 426-430. Retrieved from: http://papers.cumincad.org/cgibin/works/Show?_id=sigradi2012_368 